\title{
Efeito da prednisona em lesão medular aguda experimental em ratos
}

\author{
[Effect of prednisone on acute experimental spinal cord injury in rats] \\ C.M.O. Silva ${ }^{1}$, E.G. Melo ${ }^{2 *}$, A.E.R.F. Almeida ${ }^{2}$, M.G. Gomes ${ }^{2}$, C.H.O. Silva ${ }^{3}$, M.A. Rachid ${ }^{4}$, \\ D. Verçosa Júnior ${ }^{5}$, N.T. Vieira ${ }^{6}$, S.A. França ${ }^{7}$ \\ ${ }^{1}$ Universidade Presidente Antonio Carlos - UNIPAC - Conselheiro Lafaiete, MG \\ ${ }^{2}$ Escola de Veterinária - UFMG \\ Caixa Postal 567 \\ 30123-970 - Belo Horizonte, MG \\ ${ }^{3}$ Universidade Federal de Viçosa - Viçosa, MG \\ ${ }^{4}$ Pontifícia Universidade Católica - Betim, MG \\ ${ }^{5}$ Aluno de pós-graduação - EV-UFMG - Belo Horizonte, MG \\ ${ }^{6}$ Centro de Controle de Zoonoses - Sabará, MG \\ ${ }^{7}$ Universidade de Vila Velha - Vila Velha, ES
}

\begin{abstract}
RESUMO
Foram utilizados 25 ratos (Rattus novergicus) submetidos a trauma experimental da medula espinhal, empregando-se aparelho estereotáxico com um peso de 50,5g comprimindo a duramáter durante cinco minutos. Após o trauma, os animais foram divididos em cinco grupos de cinco. O grupo A (controle) recebeu placebo oito horas após o trauma; os grupos B, C, D e E receberam prednisona oito, 24, 48 e 120 horas após o trauma, respectivamente. A prednisona foi administrada na dose inicial de $2 \mathrm{mg} / \mathrm{kg}$, durante cinco dias, com diminuição progressiva até o $26^{\circ}$ dia. Os animais foram avaliados conforme a capacidade motora, posicionamento proprioceptivo, reflexo de localização, plano inclinado e sensibilidade dolorosa. Após 33 dias da cirurgia, foram sacrificados para avaliação histológica das medulas espinhais. Observaram-se degeneração das raízes nervosas, necrose medular, inflamação local e reação glial, sem diferenças entre os grupos. Não houve correlação entre os testes neurológicos. A utilização da prednisona na lesão medular aguda não teve efeito diferenciado com o tempo e não foi prejudicial na recuperação neurológica, mesmo quando utilizada tardiamente.
\end{abstract}

Palavras-chave: rato, prednisona, modelo experimental, lesão medular

\begin{abstract}
Twent-five rats (Rattus novergicus) were submitted to an experimental spinal cord injury by using a $50.5 \mathrm{~g}$ stereotaxic equipament to apply pressure on the duramater during five minutes. After the spinal cord injury, the animals were randomly distributed into five groups of five animals each: one group received placebo 8 hours after injury and the remaining four groups received prednisone at 8, 24, 48, and 120 hours after injury. Prednisone was applied at $2 \mathrm{mg} / \mathrm{kg}$ dosage during five days and progressively reduced until the $26^{\text {th }}$ day. Animals were evaluated by motor capacity, proprioceptive positioning, locating reflex, inclined plan, and sensibility to pain. The animals were sacrificed 33 days after surgery for histological study of the spinal cords that revealed degeneration of the rootlets, necrosis, inflammatory foci, and reactive gliosis, with no significant differences among the treated groups. The administration of high dosages of prednisone to animals with acute spinal cord injury had no deleterious effects on neurological recovery, even when used late.
\end{abstract}

Keywords: rat, prednisone, experimental models, spinal cord injury

Recebido em 21 de maio de 2007

Aceito em 14 de abril de 2008

*Autor para correspondência (corresponding author)

E-mail: eliane@vet.ufmg.br

Apoio: FAPEMIG 


\section{INTRODUÇÃO}

A lesão à medula espinhal geralmente resulta em déficits neurológicos graves e de longa duração (Tator e Fehlings, 1991; Araújo, 2005). Além de ser um dos problemas neurológicos mais comuns na prática veterinária, também é muito instigante (Braund et al., 1990). Uma terapia efetiva para tratar a medula espinhal lesada é uma das maiores indagações que desafiam médicos veterinários e humanos (Hoerlein et al., 1983).

Os danos à medula espinhal variam consideravelmente dependendo da gravidade, velocidade e duração da injúria. Um trauma agudo resulta em déficits neurológicos causados pela interrupção direta das vias neuronais, e por alterações subseqüentes que ocorrem por um período de horas até dias após a lesão primária. Esses traumas geralmente causam hemorragia, edema e isquemia e afetam principalmente a substância cinzenta. Na maioria das vezes, a agressão primária não é suficiente para causar dano permanente, mas desencadeia uma série de eventos secundários progressivos que agravam essas primeiras lesões. Mecanismos vasculares, bioquímicos e eletrolíticos são responsáveis por esses eventos pós-traumáticos, e coletivamente resultam em redução do fluxo sangüíneo espinhal, isquemia e eventual necrose (Braund et al., 1990; Bergman et al., 2000a; Lu et al., 2000; Mallei et al., 2005).

Embora não se tenha o controle sobre o trauma inicial à medula espinhal, os tratamentos clínico e/ou cirúrgico podem influenciar a sobrevivência neuronal pela interrupção dos eventos subseqüentes ao trauma. O tempo para se iniciar o tratamento é fundamental na determinação do prognóstico (Bergman et al., 2000b). Exames patofisiológicos do trauma agudo da medula espinhal mostram que a progressão dos danos teciduais já se encontra quase concluída em 24 horas, então se preconiza que o tratamento para ser efetivo deve ser instituído dentro desse prazo (Janssens, 1991).

Em razão da natureza debilitante das lesões de medula e da falta de tratamento efetivo, inúmeras pesquisas têm sido feitas para investigar o mecanismo patofisiológico e a possível interrupção das suas seqüelas (Brown e Hall, 1992). O trauma medular experimental é estudado com o propósito de se entender melhor a patologia e buscar alternativas terapêuticas mais eficientes para tratá-la (Janssens, 1991).

Os corticóides são as principais drogas utilizadas por veterinários para tratar o trauma medular, apesar de sua eficácia ser bastante questionada (Von Euler et al., 1996). Evidências sugerem que seu efeito se deve principalmente à capacidade de inibir a peroxidação de lipídios pelos radicais livres, quando empregados logo após a injúria em altas dosagens (Brown e Hall, 1992; Von Euler et al., 1996) e de aumentar o fluxo sangüíneo espinhal e a perfusão dos microvasos, melhorando clinicamente a recuperação neurológica (Tator e Fehlings, 1991).

A prednisona é um corticóide comumente utilizado no tratamento de cães com lesão de medula espinhal (Bondy e Cohn, 2002). E, contradizendo estudos publicados (Bergman et al., 2000b; Yang et al., 2003) que desencorajam o uso de corticóides tardiamente ao trauma de medula espinhal, este medicamento tem demonstrado bons resultados clínicos, mesmo quando instituído dias após a injúria ${ }^{1}$.

O objetivo desse estudo foi avaliar o efeito do tratamento com a prednisona, em dosagem alta, administrada em diferentes tempos após o trauma de medula espinhal produzido experimentalmente em ratos, por meio de estudos clínico e histológico.

\section{MATERIAL E MÉTODOS}

Foram utilizados 25 ratos machos adultos da espécie Rattus novergicus, variedade Wistar, com três meses de idade, pesando entre 272 e $377 \mathrm{~g}^{2}$. Os animais foram divididos, por meio de sorteio, em cinco grupos e colocados em caixas plásticas com cinco animais cada. Eles receberam ração comercial para roedores e água ad libitum e foram submetidos a ciclos de 12 horas com luz e 12 horas sem luz. Todos os animais foram avaliados neurologicamente segundo os testes constantes na Tab. 1, sendo que nenhum deles apresentou déficit. Os ratos foram mantidos no biotério por 14 dias para aclimatação.

${ }^{1}$ Couto - comunicação pessoal, 2006, Universidade Federal de Minas Gerais, Belo Horizonte, MG.

${ }^{2}$ Aprovado pelo Comitê de Ética em Experimentação Animal - CETEA - UFMG - protocolo $n^{\circ}$ 092/05. 
Ao final desse período, os animais foram preparados para cirurgia. Imediatamente antes da cirurgia, realizou-se antibioticoterapia com cefalotina $^{3}$, e medicação pré-anestésica com cloridrato de tramadol ${ }^{4}$. A indução e a manutenção anestésica foram realizadas com isoflurano ${ }^{5}$ fornecido por meio de máscara. Os animais foram posicionados em decúbito ventral. A incisão de pele e tecido subcutâneo foi realizada na linha média dorsal estendendo de T6 a L1. As inserções dos músculos epiaxiais foram incisadas, e eles afastados lateralmente. Com o auxílio de uma pinça goiva, realizou-se osteotomia do processo espinhal de T12 ou T13 e, em seguida, a retirada da lâmina dorsal da vértebra empregando-se drill pneumático neurológico. Após a visualização da medula espinhal, foi provocado trauma mecânico sobre a duramáter com emprego de um aparelho estereotáxico e um peso de $50,5 \mathrm{~g}$ exercendo pressão sobre a região de $\mathrm{T} 12$ ou $\mathrm{T} 13$ durante cinco minutos. Após a retirada da compressão, realizou-se a sutura dos músculos, espaço morto e pele. Os animais foram mantidos em caixa aquecida até completa recuperação anestésica e receberam analgesia com cloridrato de tramadol durante cinco dias.

Todos os animais foram submetidos ao mesmo protocolo de tratamento, mas com início em diferentes tempos após a cirurgia. A medicação foi administrada por via oral em forma de xarope $^{6}$ em volume e intervalos semelhantes, sendo que o grupo-controle (A) recebeu o xarope sem nenhuma droga, e os demais grupos (B, C, D e E) tomaram o xarope com prednisona ${ }^{7}$. No início do tratamento, a prednisona foi administrada na dose de $2 \mathrm{mg} / \mathrm{kg}$, sendo considerada uma dose alta, indicada quando se deseja imunossupressão.

Os animais foram tratados com prednisona (grupos $\mathrm{B}, \mathrm{C}, \mathrm{D}$ e E) e placebo (grupo A) nas doses de $2 \mathrm{mg} / \mathrm{kg}, 1 \mathrm{mg}$ e $0,5 \mathrm{mg} / \mathrm{kg}$, cada uma das doses foi utilizada por um período de cinco dias. As doses de $2 \mathrm{mg}$ e $\mathrm{lmg} / \mathrm{kg}$ foram administradas de 12/12 horas e a de $0,5 \mathrm{mg} / \mathrm{kg}$ de $24 / 24$ horas. Após este período

${ }^{3}$ Ariston Indústrias Químicas e Farmacêuticas Ltda - São Paulo, SP.

${ }^{4}$ Solução oral - Manipulado pela Farmácia Artesanal - Belo Horizonte, MG.

${ }^{5}$ Cristália Produtos Químicos Farmacêuticos Ltda - Itapira, SP.

${ }^{6}$ Xarope base + glicerina $10 \%$ - manipulado pela Farmácia da UFMG - Belo Horizonte, MG.

${ }^{7}$ Xarope base + glicerina $10 \%+$ prednisona $0,0125 \mathrm{mg} /$ gota manipulado pela Farmácia da UFMG - Belo Horizonte, MG.
(15 dias), foram administradas mais três doses de $0,5 \mathrm{mg} / \mathrm{kg}$ com intervalos de 48 horas. Os tratamentos iniciaram-se oito horas após a cirurgia para os grupos A e B. Para os grupos C, D e E, os tratamentos iniciaram-se 24,48 e 120 horas após a cirurgia, respectivamente.

Os animais foram submetidos à avaliação neurológica 24 horas e a cada três dias após a cirurgia até o $33^{\circ}$ dia. $\mathrm{O}$ exame neurológico consistiu na realização dos cinco testes apresentados na Tab. 1. Os animais foram testados pela mesma pessoa durante todo o período experimental.

Tabela 1. Escores para avaliação de déficit neurológico em ratos

\begin{tabular}{lr}
\hline Avaliação neurológica & Escore \\
\hline Sensibilidade dolorosa & 0 \\
Ausente & 1 \\
Diminuído & 2 \\
Normal &
\end{tabular}

Capacidade motora

- Movimento nos posteriores Ausente, sem apoio de peso Sutil, sem apoio de peso

Visível, sem apoio de peso

Apoio de peso - andar com déficit acentuado

0

Andar

Déficit moderado

ausente na barra de $7,7 \mathrm{~cm}$

Normal ou déficit discreto/presente barra $7,7 \mathrm{~cm}$

- Andar na barra de:

$6,7 \mathrm{~cm}$

$5,7 \mathrm{~cm}$

$4,7 \mathrm{~cm}$

$3,7 \mathrm{~cm}$

$2,7 \mathrm{~cm}$

$1,7 \mathrm{~cm}$

Posicionamento proprioceptivo

Ausente

Diminuído

Normal

1

Reflexo de localização

Ausente

Diminuído

Normal

1

ermanecer em plano inclinado

$10^{\circ}$ - menor que antes da cirurgia $\quad 1$

$20^{\circ}$ - menor que antes da cirurgia $\quad 2$

$30^{\circ}$ - menor que antes da cirurgia $\quad 3$

Mesmo ângulo antes e após cirurgia

4

Quanto maior o escore atingido, mais próximo da normalidade o animal se encontra. 
A sensibilidade dolorosa foi testada pela pressão de uma pinça hemostática mosquito de Halsted fechada até a primeira cremalheira na prega interdigital. A capacidade motora foi baseada na observação da atividade espontânea do animal, primeiro em um campo aberto e, quando apresentava apenas déficit discreto na deambulação, era colocado para caminhar em barras de madeira cada vez mais estreitas de acordo com a capacidade neurológica do animal. As barras tinham um metro de extensão, e a largura variava de 7,7 a $1,7 \mathrm{~cm}$, com diferença de $1 \mathrm{~cm}$ entre elas. Foi considerada para escore a menor barra em que o animal conseguia caminhar utilizando os quatro membros. $\mathrm{O}$ posicionamento proprioceptivo foi realizado flexionando-se a pata do animal de maneira que sua superfície dorsal ficasse em contato com o chão. Foram avaliadas a rapidez e a precisão com que o animal retornava a pata para a posição normal. O reflexo de localização foi verificado colocando a superfície plantar de cada membro em contato com a borda lateral de uma mesa. Foram avaliadas com que precisão e rapidez o animal colocava o membro na superfície da mesa. O teste do plano inclinado consistiu em colocar o animal em uma tábua coberta por emborrachado cuja superfície podia ser ajustada para permanecer em vários ângulos. Dessa forma, foi possível estabelecer o maior ângulo no qual o animal conseguia manter sua posição por cinco segundos.

No $33^{\circ}$ dia os animais foram sacrificados com sobredose de tiopental sódico. A medula espinhal foi retirada junto com um segmento da coluna e imersa imediatamente em paraformaldeído a $4 \%$ e, em seguida, o animal foi necropsiado. Após 30 dias, os segmentos de coluna foram dissecados para obtenção das medulas espinhais que foram processadas pela técnica rotineira de inclusão em parafina e coradas pela hematoxilina-eosina para avaliação histológica pela microscopia óptica.

As análises estatísticas foram realizadas com 0 auxílio do pacote estatístico $\mathrm{SAS}^{8}(\mathrm{P} \leq 0,05)$. Os efeitos dos tratamentos foram inicialmente estudados em cada uma das 12 avaliações. Aplicou-se o teste não-paramétrico KruskalWallis com o intuito de testar a hipótese de

${ }^{8}$ Statistical Analysis System, version 8, SAS Institute Inc., Cary, NC, USA, licenciado para a Universidade Federal de Viçosa, 2006 igualdade entre os efeitos dos tratamentos. Apesar de o número de ratos utilizados por tratamento ter sido igual a cinco, número não ideal para se utilizar a aproximação assintótica pela distribuição de qui-quadrado do teste K-W, o teste é melhor opção que a ANOVA usual. Se o teste $\mathrm{K}-\mathrm{W}$ indicasse diferença significativa entre os grupos, eles seriam comparados dois a dois, portanto, haveria 10 comparações, pelo teste não paramétrico Wilcoxon rank sum, adotar-se-ia p-valor multiplicado por 10 , corrigido por Bonferroni em cada comparação. Adicionalmente, foram calculados os valores médios dos testes dos cinco ratos de cada grupo por avaliação $(\mathrm{Y})$, para se proceder a análises de regressão $\mathrm{Y}=\mathrm{f}(\mathrm{X})$, sendo $\mathrm{X}$ as 12 avaliações. $\mathrm{O}$ objetivo das análises de regressão foi comparar as respostas aproximadas de cada um dos grupos tratados com prednisona com o grupo-controle. Ajustaram-se os modelos lineares simples de primeiro grau, $Y=\alpha+\beta_{1} . X+\varepsilon$, e também o de segundo grau, $Y=\alpha+\beta_{1} \cdot X+\beta_{2} \cdot X^{2}+\varepsilon$. Os modelos foram ajustados e comparados quanto à igualdade dos parâmetros $\alpha, \beta_{1}$ e $\beta_{2}$. A histologia foi analisada pelo método qualitativo descritivo.

\section{RESULTADOS E DISCUSSÃO}

Nenhum animal apresentou infecção da ferida cirúrgica e/ou alteração que sugerisse reação ao fio empregado. Não foram observadas mudanças de comportamento, apetite, defecação e micção.

Todos os animais apresentaram déficit neurológico de moderado a acentuado na primeira avaliação neurológica, 24 horas após a cirurgia, sendo que alguns animais foram mais acometidos do que outros. Panjabi e Wrathall (1988) também encontraram variações em lesões medulares causadas por um mesmo peso, possivelmente causadas pelo aumento da freqüência respiratória no momento do trauma, levando à movimentação da coluna, ou por variações anatômicas dos animais, tais como dimensão da medula e da massa corporal.

A avaliação neurológica foi realizada mediante utilização de vários testes comportamentais, conforme Von Euler et al. (1996) e Farooque (2000) que constataram que esses testes fornecem informações úteis e importantes para se avaliar uma lesão medular experimental, incluindo a melhora espontânea com o passar do tempo e o efeito de diferentes tratamentos. Ma et 
al. (2001) reforçam que, para avaliar a função medular residual e a recuperação funcional após uma lesão medular, em modelos roedores, são recomendados vários testes comportamentais e não apenas um.

$\mathrm{Na}$ avaliação dos exames neurológicos, não ocorreram indícios de efeitos diferenciados em nenhum dos grupos e em nenhum dos dias de avaliação pelo teste Kruskal-Wallis. Na análise de regressão, todos os testes apresentaram escores diferentes entre os dias de avaliação neurológica (Tab. 2).

Tabela 2. Teste de K-W aplicado em cada dia de avaliação

\begin{tabular}{ccc}
\hline \multirow{2}{*}{ Variáveis } & \multicolumn{2}{c}{ P-valor ${ }^{*}$ (dia de observação) } \\
\cline { 2 - 3 } & Mínimo & Máximo \\
\hline CM & $0,56(1)$ & $0,99(30)$ \\
PP & $0,29(12)$ & $0,41(* *)$ \\
LC & $0,18(27)$ & $1,00(1)$ \\
PI & $0,36(15)$ & $0,70(21)$ \\
\hline
\end{tabular}

*Com 4 graus de liberdade, Prob $\left(\chi^{2}>\right.$ valor calculado $\mathrm{K}-\mathrm{W}$ ).

**P-valores iguais para os dias $1,6,9,18,21,24,27$, 30 e 33.

$\mathrm{CM}=$ capacidade motora; $\mathrm{PP}=$ posicionamento proprioceptivo, $\mathrm{LC}=$ reflexo de localização; $\mathrm{PI}=$ plano inclinado.

$\mathrm{Na}$ análise de regressão ajustaram-se modelos de regressão completos, isto é, com parâmetros para todos os grupos, inicialmente o de primeiro grau e depois o de segundo. Para as variáveis LC e PI o modelo linear de primeiro grau apresentou melhor ajuste aos dados. Para a variável PP, o modelo de segundo grau foi o mais adequado, enquanto para $\mathrm{CM}$ ambos os modelos se ajustaram bem e optou-se pelo de primeiro grau por simplicidade. Todos os testes apresentaram uma resposta significativa de escores médios com o tempo pela análise de regressão, com $\mathrm{P} \leq 0,05$ para todos os testes $\mathrm{t}$ dos coeficientes da regressão e também para o teste $\mathrm{F}$ dos modelos de regressão. Ou seja, um mesmo teste apresentou escores significativamente diferentes entre os dias de avaliação neurológica. Os resultados resumidos das análises de regressão estão apresentados nas próximas tabelas. São indicados os modelos ajustados e os resultados dos testes de identidade de modelos, com a indicação dos grupos que apresentaram respostas iguais ao controle nas 12 avaliações.
Os testes para a capacidade motora utilizados neste experimento foram similares aos utilizados por Von Euler et al. (1996), Farooque (2000) e Thomas (2000). Foi verificado que o teste com a barra possibilitou detectar déficits mínimos, não visualizados na deambulação, eficiente para avaliar a melhora funcional do animal e com a vantagem de ter uma escala gradual objetiva. Moore (1992) também constatou que algumas vezes as anormalidades visualizadas na deambulação são bem sutis e subjetivas, e que a aplicação de outros testes neurológicos pode ajudar a percebê-las.

Para a variável capacidade motora, todos os grupos apresentaram respostas médias iguais ao controle nas 12 avaliações. Vale ressaltar que o grupo $\mathrm{E}$, que iniciou tardiamente o protocolo com prednisona, teve o melhor desempenho se for considerado $10 \%$ como nível de significância (Tab. 3). No início das avaliações, esse grupo tinha escores menores do que os outros, mas ao final esses escores se tornaram superiores (Fig. 1). Este resultado sugere um possível efeito benéfico da prednisona utilizada 120 horas após lesões medulares agudas, o que contradiz autores que relatam que a utilização de corticóides após oito horas (Bergmann et al., 2000b) ou 24 horas (Yang et al., 2003) teria efeito deletério na recuperação neurológica.

Tabela 3. Modelos de regressão ajustados para capacidade motora (CM), com respectivos testes de igualdade dos parâmetros, para os grupos B, C, D e E comparados com $\mathrm{A} . \mathrm{R}^{2}=91,93 \%, \mathrm{P}<0,0001$ para o teste $\mathrm{F}$ do modelo completo com 9 e 50 graus de liberdade, $\mathrm{F}=63,31$

\begin{tabular}{ccc}
\hline \multirow{2}{*}{ Grupos } & \multicolumn{2}{c}{$\mathrm{CM}=\alpha+\beta_{1} \cdot$ dia } \\
\cline { 2 - 3 } & $\alpha$ & $\beta_{1}$ \\
\hline A & 2,050 & 0,194 \\
B & $(=)$ & $(=)$ \\
C & $(=)$ & $(=)$ \\
D & $(=)$ & $(=)$ \\
E & $1,003^{7,2 \%}$ & $0,251^{5,6 \%}$ \\
\hline
\end{tabular}

$(=)$ :indica que $\mathrm{o}$ teste $\mathrm{t}$ não rejeitou $(\mathrm{P}>0,05)$ a hipótese de igualdade entre os parâmetros do modelo com o do grupo controle (A). Estimativas foram apresentadas somente quando o teste indicou diferença significativa.

Após a lesão medular experimental todos os animais apresentaram déficit proprioceptivo, e nenhum recuperou totalmente essa função. Este dado é condizente com Moore (1992) e Thomas (2000) que afirmaram que a propriocepção é o primeiro parâmetro neurológico a ser afetado, logo no início das doenças neurológicas, e que, na recuperação, é o último a retornar à normalidade. 


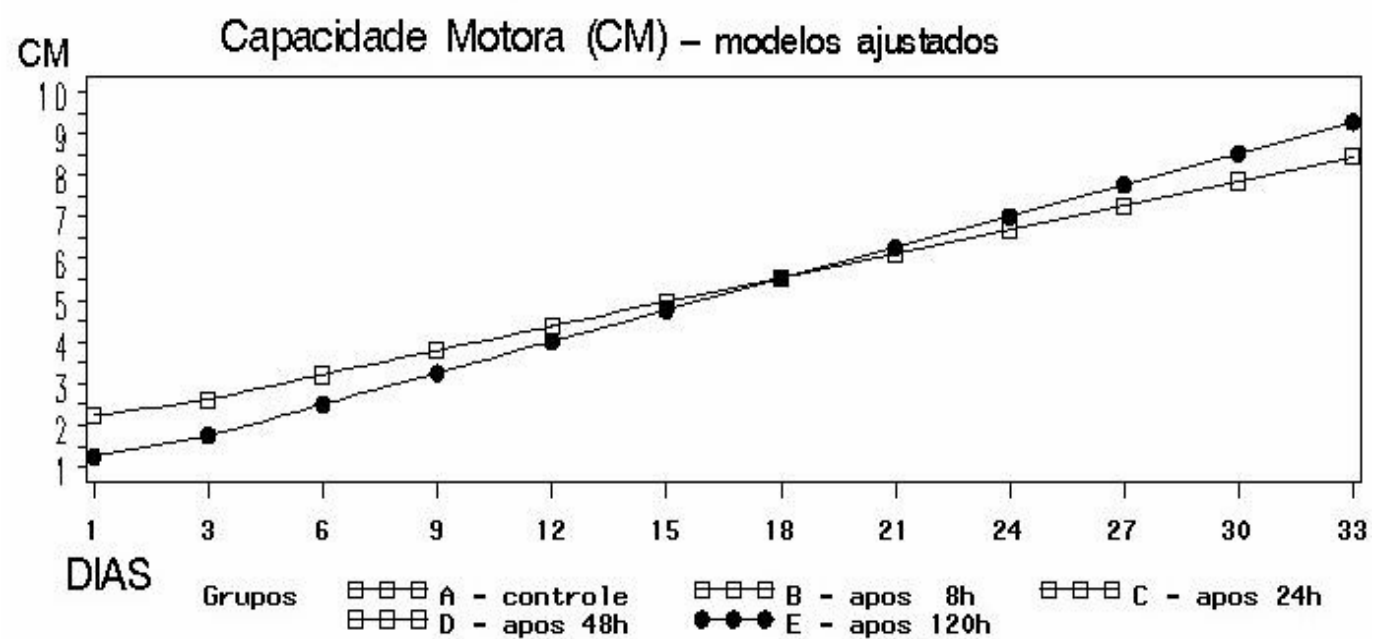

Figura 1. Modelos ajustados mostrando a evolução da capacidade motora dos grupos em cada avaliação. Os grupos A, B, C e D estão representados por uma única linha, pois têm escores idênticos. Se for adotado 10\% como nível de significância, o grupo E teria uma resposta aproximada diferente do controle.

Em relação ao posicionamento proprioceptivo, os grupos $\mathrm{A}, \mathrm{C}$ e $\mathrm{E}$ apresentaram respostas médias aproximadas iguais nas 12 avaliações. $\mathrm{O}$ grupo $\mathrm{B}$ apresentou somente uma constante de regressão diferente do controle no primeiro dia de avaliação, mas a evolução foi similar aos grupos A, C e E. Já o grupo D apresentou uma resposta diferente do controle (Tab. 4). Este último grupo teve recuperação melhor do que os demais (Fig. 2), apontando possível ação benéfica da prednisona quando utilizada 48 horas após a injúria. Este resultado não era esperado, pois segundo Bergman et al. (2000b), Lu et al. (2000), Bondy e Cohn (2002) e Lee et al. (2005) os corticóides só teriam efeito benéfico na reabilitação quando utilizados até oito horas após o trauma. Outra possibilidade, é que esta ocorrência talvez possa ser atribuída à diferença individual entre os animais e ao pequeno número de animais em cada grupo.
No teste do reflexo de localização, verificou-se que os grupos B e D apresentaram uma resposta média igual ao controle $\mathrm{A}$, a resposta do grupo $\mathrm{E}$ diferiu apenas por uma constante, mas com evolução igual ao longo do tempo, enquanto o grupo $\mathrm{C}$ apresentou uma resposta estatisticamente diferente (Tab. 5). O grupo C, que obteve os menores escores na primeira avaliação, foi o que apresentou os maiores escores na última avaliação neurológica (Fig. 3). $\mathrm{O}$ melhor desempenho dos ratos do grupo $\mathrm{C}$ sugere um potencial efeito benéfico da prednisona iniciada 24 horas após a lesão. Este resultado difere de Von Euler et al., 1996; Lu et al., 2000 e Yang et al., 2003 que indicaram a utilização de corticóides o mais rápido possível após a injúria para se obter um maior efeito benéfico.

Tabela 4. Modelos de regressão ajustados para posicionamento proprioceptivo (PP), com respectivos testes de igualdade dos parâmetros, para os grupos B, C, D e E comparados com A. R ${ }^{2}=93,55 \%$, $\mathrm{P}<0,0001$ para o teste $\mathrm{F}$ do modelo completo com 14 e 45 graus de liberdade, $\mathrm{F}=46,66$

\begin{tabular}{cccc}
\hline \multirow{2}{*}{ Grupos } & \multicolumn{3}{c}{$\mathrm{PP}=\alpha+\beta_{1} \cdot \operatorname{dia}+\beta_{2} \cdot$ dia $^{2}$} \\
\cline { 2 - 4 } & $\alpha$ & $\beta_{1}$ & $\beta_{2}$ \\
\hline A & $-0,158$ & 0,085 & $-0,002$ \\
B & 0,085 & $=$ & $=$ \\
C & $=$ & $=$ & $=$ \\
D & 0,206 & 0,051 & 0,001 \\
E & $=$ & $=$ & $=$ \\
\hline
\end{tabular}

$(=)$ : indica que o teste t não rejeitou $(\mathrm{P}>0,05)$ a hipótese de igualdade entre os parâmetros do modelo com o do grupo controle (A). Estimativas foram apresentadas somente quando o teste indicou diferença significativa. 


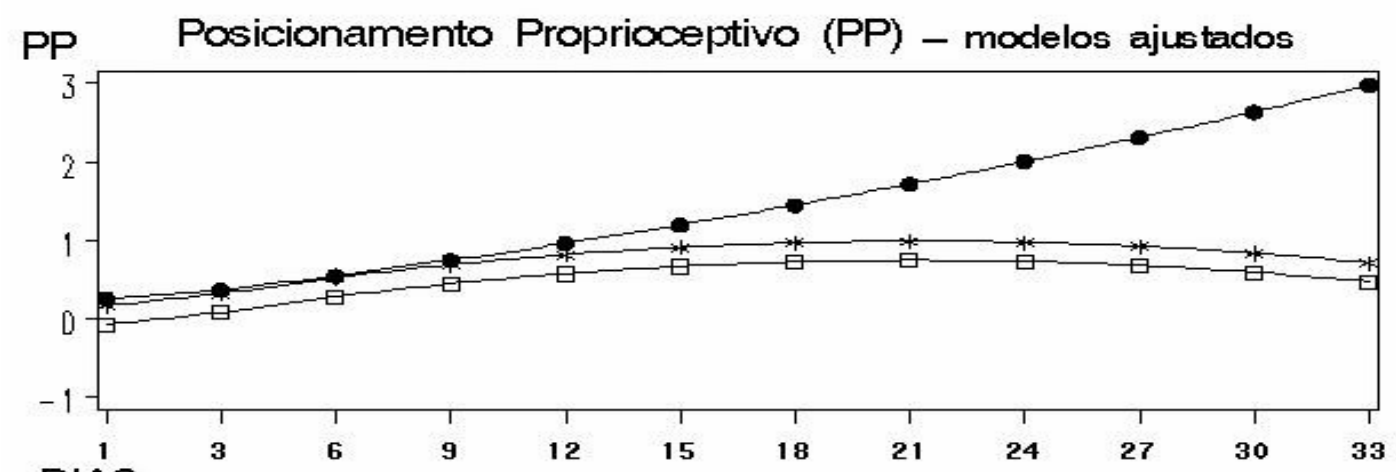

DIAS

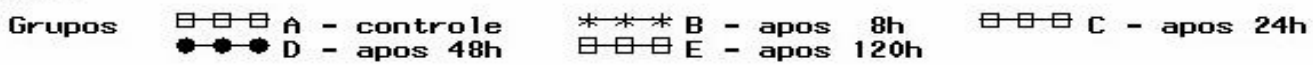

Figura 2. Modelo ajustado mostrando a evolução do posicionamento proprioceptivo dos grupos em cada avaliação. Grupos A, C e E apresentaram modelos ajustados idênticos e estão representados por uma única linha. O modelo ajustado para o grupo B apresentou somente uma constante diferente do controle. O grupo D apresentou resposta estatisticamente diferente do controle.

Tabela 5. Modelos de regressão ajustados para reflexo de localização (LC), com respectivos testes de igualdade dos parâmetros, para os grupos $\mathrm{B}, \mathrm{C}, \mathrm{D}$ e $\mathrm{E}$ comparados com $\mathrm{A} . \mathrm{R}^{2}=$ $85,09 \%, \mathrm{P}<0,0001$ para o teste $\mathrm{F}$ do modelo completo com 9 e 50 graus de liberdade, $\mathrm{F}=$ 31,70

\begin{tabular}{ccc}
\hline \multirow{2}{*}{ Tratamentos } & \multicolumn{2}{c}{$\mathrm{LC}=\alpha+\beta_{1}$.dia } \\
\cline { 2 - 3 } & $\alpha$ & $\beta_{1}$ \\
\hline A & 0,006 & 0,033 \\
B & $=$ & $=$ \\
C & $-0,363$ & 0,051 \\
D & $=$ & $=$ \\
E & $-0,300$ & $=$ \\
\hline
\end{tabular}

$(=)$ : indica que o teste $\mathrm{t}$ não rejeitou $(\mathrm{P}>0,05)$ a hipótese de igualdade entre os parâmetros do modelo com o do grupo controle (A).
O teste do plano inclinado, desenvolvido por Rivlin e Tator (1977), é amplamente utilizado para avaliação do trauma medular (Von Euler et al., 1996). Entretanto, Von Euler et al. (1996) consideraram que a diferença de ângulo observada entre um rato normal e outro com lesão intensa é muito pequena, apenas cerca de $25^{\circ}$, o que dificulta as correlações dos ângulos com as lesões. Segundo esse teste, a recuperação neurológica foi semelhante em todos os grupos, sendo que a resposta média do grupo B diferiu do A por um termo constante, mas sem diferença na evolução (Tab. 6, Fig. 4).

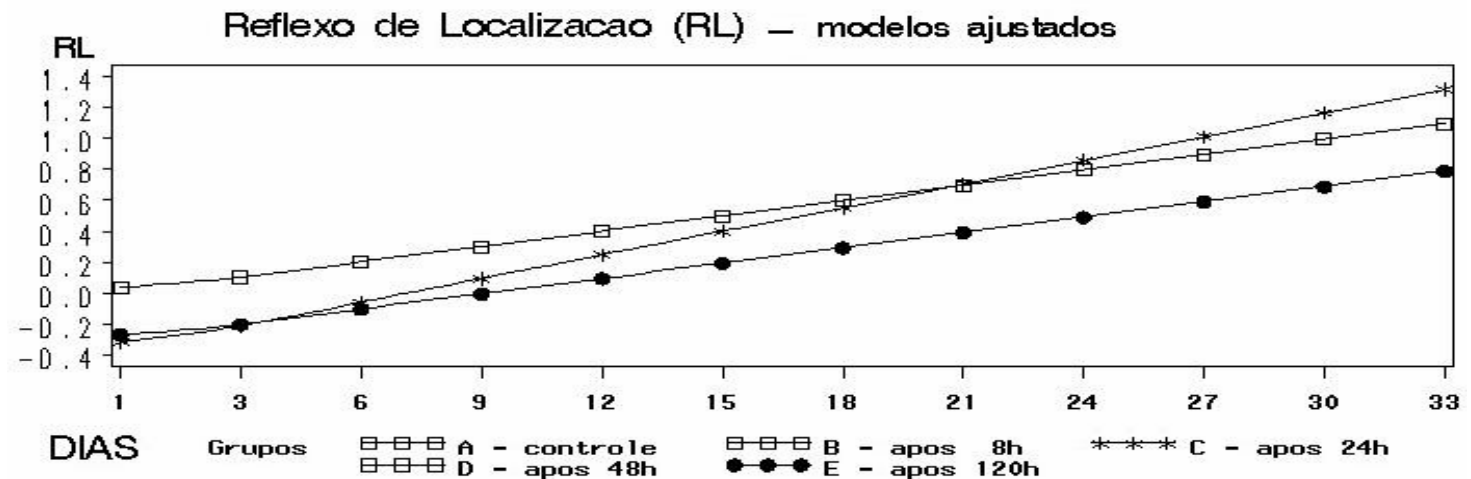

Figura 3. Modelos ajustados mostrando a evolução do reflexo de localização dos grupos em cada avaliação. Os grupos B e D apresentaram resposta média igual ao controle (A), sendo os três representados pela mesma linha. O grupo $\mathrm{E}$ diferiu apenas por uma constante, porém com igual incremento linear (retas paralelas). O grupo $\mathrm{C}$ apresentou resposta estatisticamente diferente. 
Tabela 6. Modelos de regressão ajustados para plano inclinado (PI), com respectivos testes de igualdade dos parâmetros, para os grupos B, C, D e E comparados com $\mathrm{A}$. $\mathrm{R}^{2}=70,19 \%, \mathrm{P}<0,0001$ para o teste $\mathrm{F}$ do modelo completo com 9 e 50 graus de liberdade, $\mathrm{F}=13,08$

\begin{tabular}{ccc}
\hline \multirow{2}{*}{ Grupos } & \multicolumn{2}{c}{$\mathrm{PI}=\alpha+\beta_{1}$.dia } \\
\cline { 2 - 3 } & $\alpha$ & $\beta_{1}$ \\
\hline A & 3,134 & 0,030 \\
B & 3,548 & $=$ \\
C & $=$ & $=$ \\
D & $=$ & $=$ \\
E & $=$ & $=$
\end{tabular}

$(=)$ : indica que o teste $\mathrm{t}$ não rejeitou $(\mathrm{P}>0,05)$ a hipótese de igualdade entre os parâmetros do modelo com o do grupo controle (A). Estimativas foram apresentadas somente quando o teste indicou diferença significativa.

A sensibilidade dolorosa se manteve inalterada durante todo o experimento em todos os animais e por esse motivo não foi necessário submetê-la à análise estatística. Esse fato era esperado e desejado, pois objetivou-se provocar uma lesão medular moderada e reversível. Segundo Moore (1992) e Bergman et al. (2000a), é necessário um trauma grave na medula espinhal para interromper a sensação de dor profunda e que, quando isso ocorre, o prognóstico é desfavorável. Esses dados são condizentes com os resultados encontrados, pois nenhum animal teve perda da dor profunda, e todos eles tiveram alguma recuperação neurológica.

Era esperado que o grupo $\mathrm{B}$, que iniciou o tratamento mais rapidamente após o trauma, tivesse o melhor desempenho, entretanto isso não ocorreu; em todos os grupos, a evolução foi igual ao longo do tempo. Não foi verificada correlação entre os testes, pois em cada teste um grupo obteve melhor resposta. Apesar de preconizado que os corticóides devem ser utilizados o mais rápido possível após o trauma, os resultados deste experimento indicam que a prednisona administrada após 24, 48 ou 120 horas da lesão não prejudica a recuperação neurológica. A recuperação semelhante, nos grupos tratados, indica que a prednisona não interferiu no restabelecimento. De acordo com Jeffery e Blackmore (1999) quando a lesão medular não é grave o suficiente para causar alterações permanentes, pode ocorrer recuperação espontânea, e isso pode ser observado também no tratamento placebo ou conservativo após uma lesão experimental aguda. Nestes casos os animais recuperariam suas funções com ou sem tratamento e ficariam sujeitos apenas às alterações individuais.

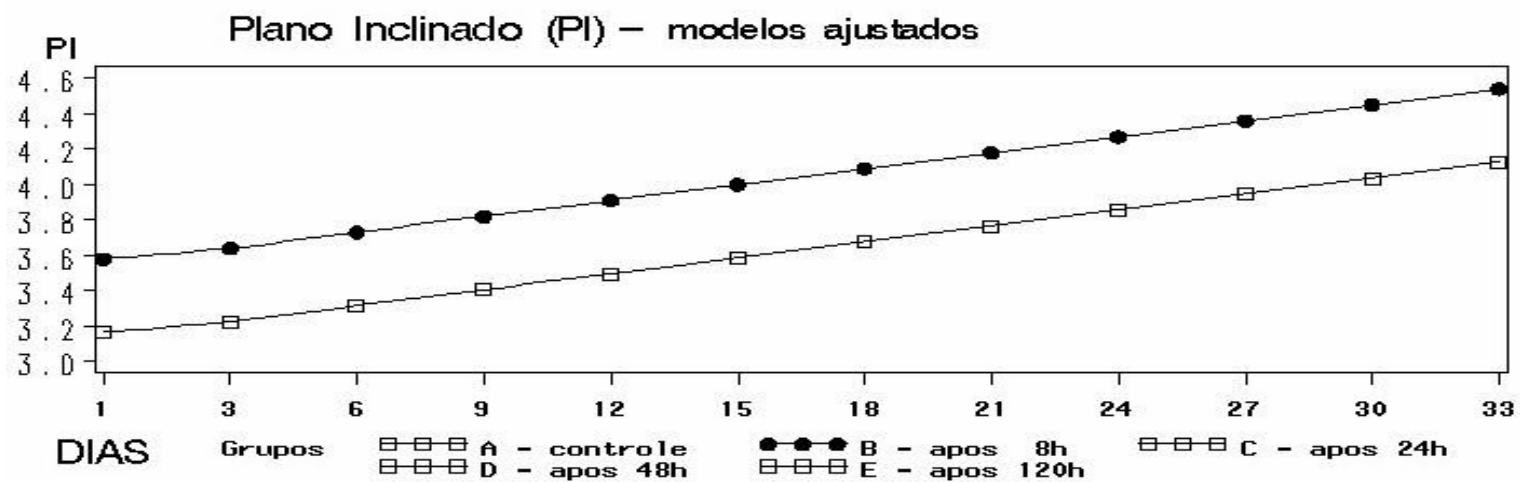

Figura 4. Modelos ajustados mostrando a evolução dos grupos no teste do plano inclinado em cada avaliação. Visualiza-se pelo gráfico que o grupo B diferiu dos demais grupos, representados por uma única linha, apenas por uma constante, mas com incremento linear igual (retas paralelas).

Em todos os animais foram encontradas alterações microscópicas semelhantes com intensidades diferentes sem, contudo, predominância de lesões mais ou menos intensas em um grupo específico.
O método de fixação da medula com paraformoldeído a $4 \%$ por 30 dias não foi eficiente, ocorreu dissolução do material, o que dificultou o seu processamento e algumas vezes com artefatos que alteraram a morfologia histológica do corte em quase toda a lâmina. 
Dessa forma, foi possível observar as alterações, mas não foi possível quantificar a área lesada.

Observou-se em todas as lâminas degeneração axonal discreta a intensa em uma ou mais raízes nervosas. Na maioria dos animais, independente do grupo, a medula espinhal apresentava cavitação contendo células de Gitter, gliócitos reativos, células inflamatórias mononucleares e vasos sangüíneos neoformados. A extensão da área de necrose variou desde um pequeno foco até cerca de um terço da medula, sendo que muitas vezes o acometimento foi mais intenso em um dos lados. Houve o envolvimento das duas substâncias, sendo que a cinzenta foi a principal afetada em extensão e intensidade, o que já foi citado por Bergman et al. (2000a) e Mallei et al. (2005) quando relatam que essa substância é mais sensível ao trauma agudo, sendo principalmente afetada nesse tipo de injúria.

Apesar de a substância cinzenta ter sido a principal afetada, a recuperação da locomoção provavelmente se deveu ao fato de a lesão ter ocorrido em $\mathrm{T} 12$ ou T13, área onde essa substância não é essencial para a enervação dos membros (Moore, 1992; Jeffery e Blackmore, 1999). Nessa região, o acometimento da substância branca é mais prejudicial à função motora por interferir na condução do impulso a partir de estruturas supraespinhais (Moore, 1992).

Os resultados encontrados na histologia estão de acordo com Janssens (1991) que relatou que a fagocitose, iniciada poucos dias após a lesão, resulta na formação de cavidades e que uma semana após o trauma todas as células da área necrótica contêm citoplasmas com aspecto espumoso. Os achados histológicos também condizem com Lu et al. (2000) que afirmaram que a lesão resultante de diferentes tipos de trauma medular agudo é freqüentemente similar, com cavitações da substância cinzenta e acometimento variável do tecido periférico, e que a extensão em que ocorre morte de neurônios e células da glia, durante os eventos secundários, é responsável pela formação de cavitações.

Não foram observadas diferenças histológicas entre os grupos, o que inviabilizou determinar se a administração de altas doses de prednisona, em diferentes tempos, promoveu alterações morfológicas distintas na medula espinhal após o trauma. Esta impossibilidade pode ter sido decorrente da falha na fixação do material.

\section{CONCLUSÕES}

A utilização de altas doses de prednisona em lesão medular aguda experimental não tem efeito diferenciado com o tempo em que é aplicada, sendo que a administração tardia não tem efeito prejudicial à recuperação neurológica. A ocorrência de recuperação espontânea em ratos com lesões medulares moderadas dificulta a avaliação do efeito do tratamento.

\section{REFERÊNCIAS BIBLIOGRÁFICAS}

ARAÚJO, I.S. Presence of free radicals in spinal cord injury. Experimental evidence in rats. Rev. Bras. Neurol., v.41, p.23-29, 2005.

BERGMAN, R.; LANZ, O.; SHELL, L. Acute spinal cord trauma: mechanisms and clinical syndromes. Vet. Med., v.95, p.846-849, 2000a.

BERGMAN, R.; LANZ, O.; SHELL, L. A review of experimental and clinical treatments for acute spinal cord injury. Vet. Med., v.95, p.855-866, 2000b.

BONDY, P.J.; COHN, L.A. Choosing an appropriate glucocorticoid treatment plan. Vet. Med., v.97, p.841-849, 2002.

BRAUND, K.G.; SHORES, A.; BRAWNER, W.R. The etiology, pathology, and pathophysiology of acute spinal cord trauma. Vet. Med., v.85, p.684-691, 1990.

BROWN, S.A.; HALL, E.D. Role of oxygenderived free radicals in the pathogenesis of shock and trauma, with focus on central nervous system injuries. J. Am. Vet. Med. Assoc. v.200, p.18491858, 1992.

FAROOQUE, M. Spinal cord compression injury in the mouse: presentation of a model including assessment of motor dysfunction. Act. Neurop., v.100, p.13-22, 2000.

HOERLEIN, B.F.; REDDING, R.W.; HOFF, E.J. et al. Evaluation of dexamethasone, DMSO, mannitol and solcoseryl in acute spinal cord trauma. J. Am. Anim. Hosp. Assoc., v.19, p.216226, 1983. 
JANSSENS, L.A.A. Mechanical and pathophysiological aspects of acute spinal cord trauma. J. Small A. Pract., v.32, p.572-578, 1991.

JEFFERY, N.D.; BLAKEMORE, W.F. Spinal cord injury in small animals 1 . Mechanisms of spontaneous recovery. Vet. Rec., v.144, p.407413, 1999.

LEE, B.H.; LEE, K.H.; YOON, D.H. et al. Effects of methylprednisolone on the neural conduction of the motor evoked potentials in spinal cord rats. J. Kor. Med. Sci., p.132-138, 2005.

LU, J.; ASHWELL, K.W.S.; WAITE, P. Advances in secondary spinal cord injury: Role of apoptosis. Spine, v.25, p.1859-1866, 2000.

MA, M.; BASSO, D.M.; WALTERS, P. et al. Behavioral and histological outcomes following graded spinal cord contusion injury in the C57BI/6 mouse. Exp. Neurol., v.169, p.239-254, 2001.

MALLEI, A.; ADEN, S.A.; BACHIS, A. et al. The nitrosteroid NCX 1015, a prednisolone derivative, improves recovery of function in rats after spinal cord injury. Brain Res., v.1062, p.1625, 2005.
MOORE, M.P. Approach to the patient with spinal disease. Vet. Clin. N. Am.: Small A. Prac., v.22, p.751-781, 1992.

PANJABI, M.M.; WRATHALL, J.R. Biomechanical analysis of experimental spinal cord injury and functional loss. Spine, v.13, p.1365-1370, 1988.

RIVLIN, A.S.; TATOR, C.H. Objective clinical assessment of motor function after experimental spinal cord injury in the rat. J. Neuros., v.47, p.577-581, 1977.

TATOR, C.H.; FEHLINGS, M.G. Review of the secondary injury theory of acute spinal cord trauma with emphasis on vascular mechanisms. J. Neuros., v.75, p.15-26, 1991.

THOMAS, W.B. Initial assessment of patients with neurologic dysfunction. Vet. Clin. N. Am.: Small A. Pract., v.30, p.1-23, 2000.

VON EULER, M.; AKESSON, E.; SAMUELSSON, E.B. et al. Motor Performance score: a new algorithm for accurate behavioral testing of spinal cord injury in rats. Exp. Neurol., v.137, p.242-254, 1996.

YANG, J.W.; JEONG, S.M.; SEO, K.M. et al. Effects of corticosteroid and electroacupuncture on experimental spinal cord injury in dogs. $J$. Vet. Sci., v.4, p.97-101, 2003. 